\title{
Hh pathway expression in human gut tissues and in inflammatory gut diseases
}

\author{
Corinne M Nielsen ${ }^{1}$, Jerrell Williams ${ }^{1}$, Gijs R van den Brink ${ }^{2}$, Gregory Y Lauwers ${ }^{1}$ \\ and Drucilla J Roberts ${ }^{1}$ \\ ${ }^{1}$ Department of Pathology, Massachusetts General Hospital, Boston, MA, USA and ${ }^{2}$ Department of \\ Experimental Internal Medicine, Academic Medical Center, Amsterdam, The Netherlands
}

\begin{abstract}
Sonic hedgehog (Shh) directs early gut patterning via epithelial-mesenchymal signaling and remains expressed in endoderm-derived tissues into the adult period. In human adult gut epithelium SHH/SHH expression is strongest in basal layers, which suggests that SHH may function in the maintenance of gut epithelial stem or progenitor cells. Recent publications suggest a role for aberrant SHH/SHH expression in gut epithelial neoplasias. We hypothesized that the regenerating gut epithelium in inflammatory gut disorders would show an upregulation of SHH/SHH signaling and this abnormal signal may explain the increased incidence of neoplasia in these diseases. Archived healthy gut and inflammatory gut diseased tissues were analyzed by RNA in situ hybridization and immunohistochemistry to describe location and levels of SHH signaling. We show that SHH/SHH and its receptor PTCH1/PTCH1 expression is restricted to the glandular epithelium of the gut, in an antiluminal pattern (strongest in basal layers and weak to absent in luminal epithelium). Inflammatory diseases of the gut show dramatic increases in epithelial SHH signaling. Expression increases in inflamed glandular epithelium (including metaplastic glandular epithelium), losing its radial (crypt-villous) polarity, and expression appears upregulated and present in all epithelial cells. We also describe strong SHH/SHH and PTCH1/PTCH1 expression in intraepithelial and mucosal inflammatory cells. We suggest that SHH signaling in inflammatory diseases of the gut acts to ensure stem cell restitution of damaged mucosal epithelium. However, such signaling may also present a risk for neoplastic transformation.
\end{abstract}

Laboratory Investigation (2004) 84, 1631-1642, advance online publication, 25 October 2004; doi:10.1038/labinvest.3700197

Keywords: $\mathrm{SHH} / \mathrm{SHH}$; PTCH1/PTCH1; inflammatory gut disease; epithelial-mesenchymal signaling; Hh pathway

Hedgehog (Hh) signaling is a fundamental pathway directing patterning events during embryogenesis. The Hh signaling pathway plays a key role in important epithelial-mesenchymal interactions in many different anatomic sites including the gastrointestinal system (gut). ${ }^{1-12}$ During gut development, Hh proteins are secreted by the epithelial cells throughout the gut and are believed to function in the underlying mesenchyme to activate molecules important in mesodermal differentiation..$^{1,2,5,13-15}$ In the developing vertebrate gut, two Hh proteins are expressed: Sonic Hh (Shh) and Indian Hh (Ihh), with Shh expressed in earlier developmental stages. ${ }^{2,7}$ Expression of the receptors/effectors for

Correspondence: Dr DJ Roberts, MD, Department of Pathology, Massachusetts General Hospital, 32 Fruit Street, Boston, MA 02114, USA.

E-mail: djroberts@partners.org

Received 22 July 2004; revised 11 September 2004; accepted 15 September 2004; published online 25 October 2004
Hh signaling, Patched (Ptc), Smoothened (Smo), and Gli denotes the location of action of the $\mathrm{Hh}$ signal. ${ }^{16,17}$ These receptors and targets are expressed in the mesenchyme subjacent to the Hh expressing endoderm during early development. ${ }^{15}$

The Hh receptor is a complex of two proteins: Ptc and Smo. In the absence of Hh protein binding, Ptc (a membrane bound protein) inhibits Smo-directed activation of downstream targets. Hh binding to Ptc releases the antagonism of Smo and allows transcriptional activation of target genes (eg Gli, Wnt, and Bmp, see Cohen ${ }^{18}$ for a review).

Shh as a signal to the mesoderm is evidenced by overexpression studies in which over/misexpression of Shh results in a mass-like hypertrophy of the gut mesoderm. ${ }^{19}$ Shh signals must have an epithelial function as well, whether direct or indirect, as ectopic expression of Shh affects both the mesoderm and epithelium in the developing gut. For example, in the developing pancreas, Shh/Shh expression is absent in the endoderm that forms the pancreatic buds. ${ }^{20}$ Ectopic expression of Shh in this 
endodermal region results in abnormal intestinallike mesenchymal differentiation of the pancreas ${ }^{13}$ and a preneoplastic intestinal alteration in the duct epithelium. ${ }^{21}$

Recently, there has been evidence that the $\mathrm{Hh}$ pathway functions in human adult gut epithelium. It has been shown that $\mathrm{SHH}$ protein expression correlates with the differentiation of gastric glands in adult human and rodent stomachs. ${ }^{22} \mathrm{SHH}$ protein expression is also detected in gastric metaplasias in the human adult gastrointestinal tract. ${ }^{22}$ It has recently been reported that epithelial malignancies of the adult gut are associated with abnormal SHH/ SHH expression. ${ }^{21,23}$ These studies have suggested that SHH may function as a mediator of gut stem cell maintenance. An unregulated progenitor cell proliferation induced via abnormal SHH protein expression or pathway activation has been suggested to play a role in all cancers of the gastrointestinal tract. ${ }^{21,23,24}$ The pathway activation has been described in a variety of epithelial carcinomas from other sites including lung and skin. ${ }^{17,25-29}$ It is thought as well that $\mathrm{Hh}$ signaling ensures that a progenitor or stem cell population is retained in these tissues. ${ }^{29-34}$

Hh signaling may be important in other human diseases involving chronic tissue injury. Hh signaling has been shown to be upregulated in chronic pulmonary fibrosis, a non-neoplastic pulmonary disease. ${ }^{35}$ Chronic pulmonary fibrosis is a fatal interstitial lung disease of multiple etiologies sharing destructive pulmonary fibrosis with associated interstitial inflammation. ${ }^{36,37}$ The Hh signal upregulated in this disease was recently shown to be derived from the infiltrating lymphocytes but was also detected in the pulmonary epithelium in injured lung regions and in circulating $\mathrm{T}$ lymphocytes. ${ }^{35}$ This is interesting as Hh signaling is active in the development of human lymphocytes. ${ }^{38-42}$ It has been suggested that signaling between the CD4 expressing lymphocytes and the injured epithelium may be associated with either the disease process or the tissue-mediated recovery. ${ }^{35}$

These findings suggested to us that the $\mathrm{Hh}$ signaling pathway may be upregulated in other tissues disturbed by chronic inflammation including those of the gastrointestinal tract, and that they may play a role in epithelial regeneration. Chronically elevated Hh signaling may increase the risk for malignancy known to be associated with many of these disorders. We elected to examine the role of $\mathrm{Hh}$ signaling in chronic inflammatory disorders of the gastrointestinal tract, including foregut inflammatory conditions such as Barrett's esophagus and chronic gastritis, and hindgut inflammatory bowel diseases such as ulcerative colitis and Crohn's disease by analyzing the mRNA and protein expression patterns of $\mathrm{SHH}$ and PTCH1 (human Ptc) and comparing them with expression in normal healthy adult and fetal gut tissues.

\section{Materials and methods}

\section{In Situ Hybridization and Immunohistochemistry}

Cases and controls were pulled by diagnosis retrieval using SNOMED search criteria from the Department of Pathology archives at Massachusetts General Hospital and personal collections of GY, both with IRB approval. Only samples from 20002003 were used for this study. The diagnostic H\&E slides were reviewed for confirmation of the diagnosis by GYL and DJR. The best representative slides from diseased and nondiseased tissue were chosen for each individual case. Nondiseased tissue sections were used as controls. In the case of ulcerative colitis, proximal small intestinal resection margins were available as normal control tissue. At least three cases and three controls were studied for each diagnosis and anatomic region. No other patient information was obtained (confidentiality was ensured). Paraffin-embedded tissue was gathered and sectioned at $3-6 \mu \mathrm{m}$ onto coated slides (Fisher) and kept at $4{ }^{\circ} \mathrm{C}$ until used. All slides were used within 3 months of sectioning. ${ }^{43}$ In situ hybridization was performed in accordance with a published protocol for paraffin sections. ${ }^{44}$ Probes for human $S H H$ and PTCH1 were obtained from C Tabin and have been published previously. ${ }^{45,46}$ The signal was detected with BM Purple AP substrate following the manufacturer's recommendations (Roche). Immunohistochemistry studies followed standard protocol with minor changes. Antigen unmasking was achieved by boiling sections in $0.01 \mathrm{M}$ sodium

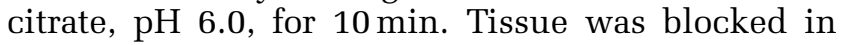
$10 \%$ sheep serum, and additional blocking of endogenous avidin/biotin binding sites was performed with an avidin/biotin blocking kit (Vector Labs). Antibodies were applied at the following dilutions: CD4 (Novocastra, 1:20), CD8 (DAKO, 1:100), CD20 (Neomarkers, 1:50), CD34 (Neomarkers, 1:50), PTCH1 (Santa Cruz, 1:50), SHH (Santa Cruz, 1:50). Protein was visualized with DAB chromagen (Sigma).

\section{Results}

All detected SHH and PTCH1 mRNA and protein expression patterns were cytoplasmic as has been described by others. ${ }^{21,22,24}$

\section{SHH Expression is Present in Normal Adult Human Gut Epithelium}

We have previously studied the expression of $\mathrm{SHH}$ (mRNA)/SHH (protein) in the normal adult human gut, where we described that SHH/SHH was not detected in the normal adult esophageal squamous epithelium or in the [corpus] or pyloric regions of the stomach. Herein, in resected surgical specimens we studied the expression of SHH/SHH in normal, noninflamed tissues from the same patients as the 
Normal Adult Tissues

SHH

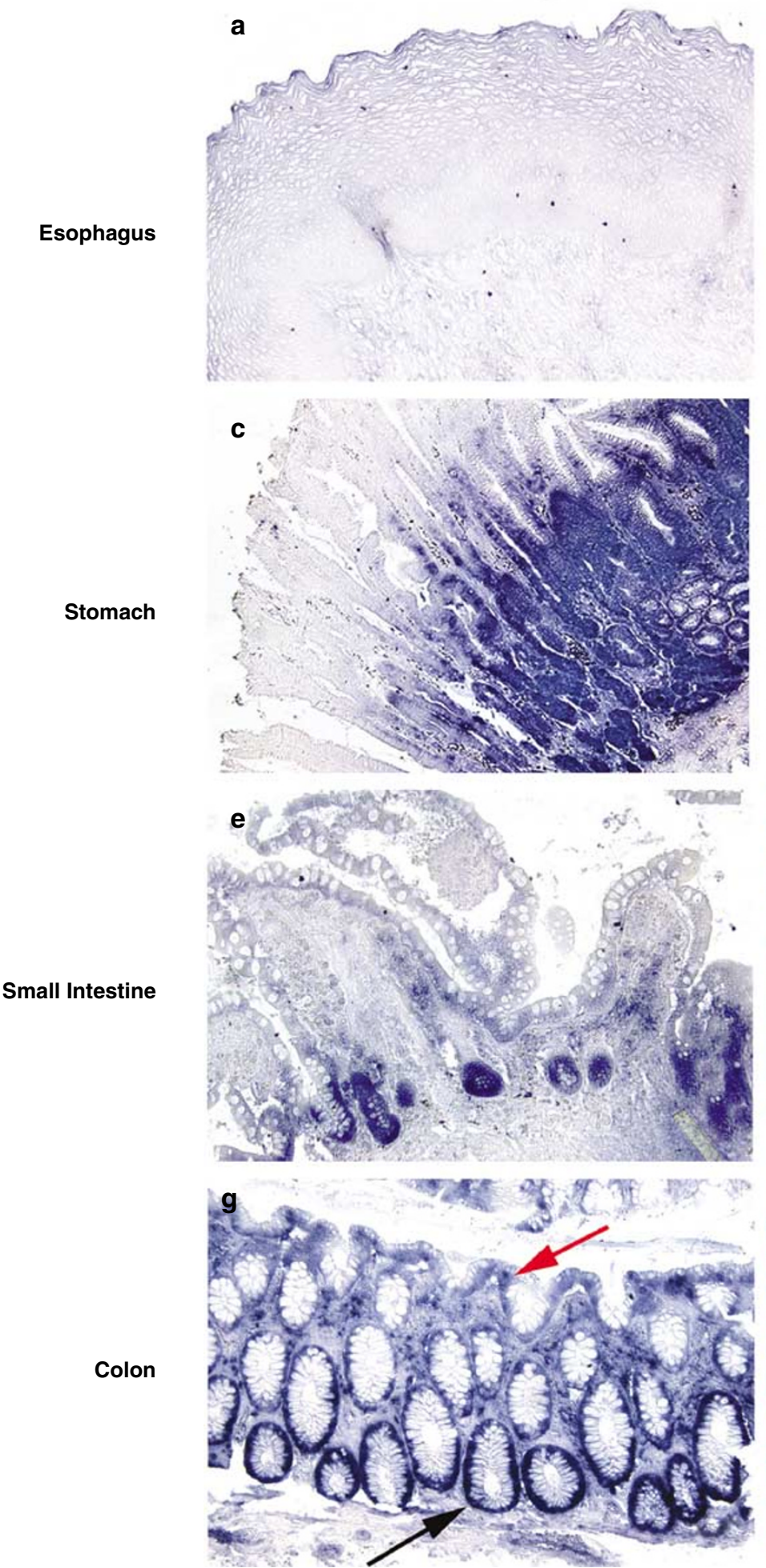

SHH
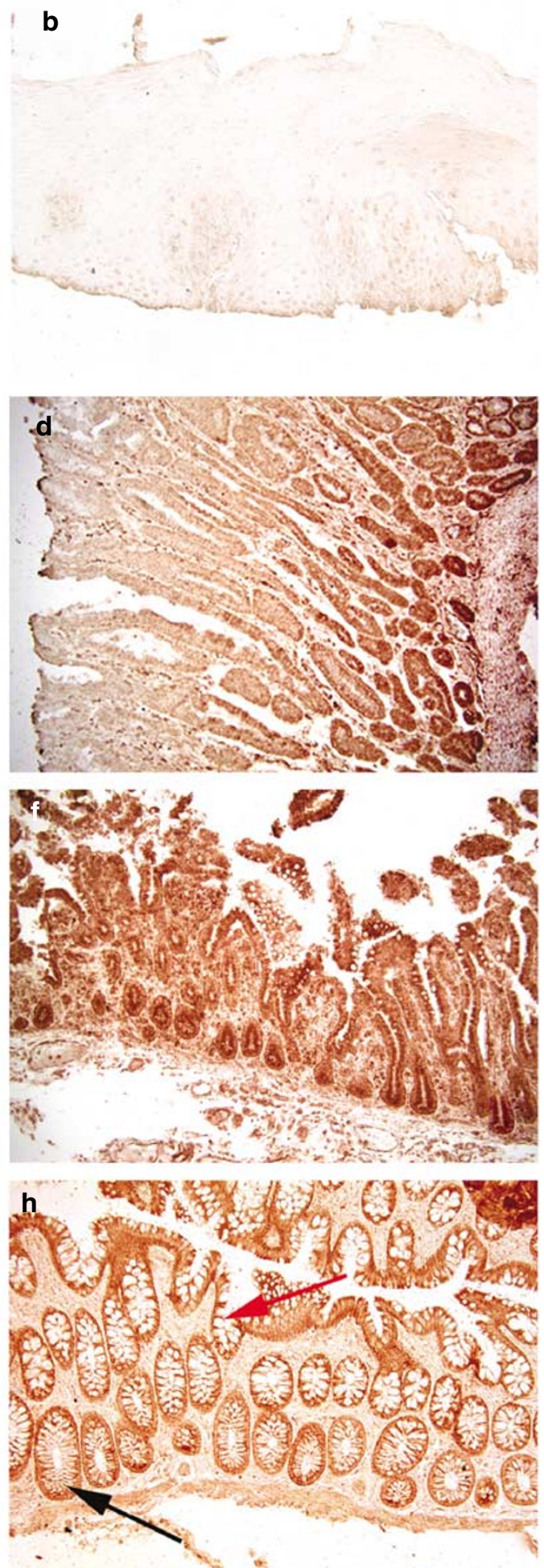

Figure 1 SHH expression in normal adult human gut. mRNA (a, $\mathbf{c}, \mathbf{e}, \mathbf{g})$ and protein $(\mathbf{b}, \mathbf{d}, \mathbf{f}, \mathbf{h})$ expression of SHH in normal adult human gut tissues. Expression is not detected in the normal $(\mathbf{a}, \mathbf{b})$ esophageal squamous epithelium. The normal stomach expresses SHH in the glands (c, d). Normal small intestine (duodenum, jejunum, and ileum) expresses SHH strongly at the base of the villi (arrow in e) without detectable $S H H$ expression in the villous tips (arrowhead in e). SHH is present in both villous base and villous tip epithelial sites (f) although stronger in the base of the villi. Healthy adult colon expresses SHH and SHH in the crypts (black arrows in $\mathbf{h}$ and g) with weak focal protein expression in the luminal epithelium (h and $\mathbf{g}$, red arrows). 


\section{Normal Adult Tissues}
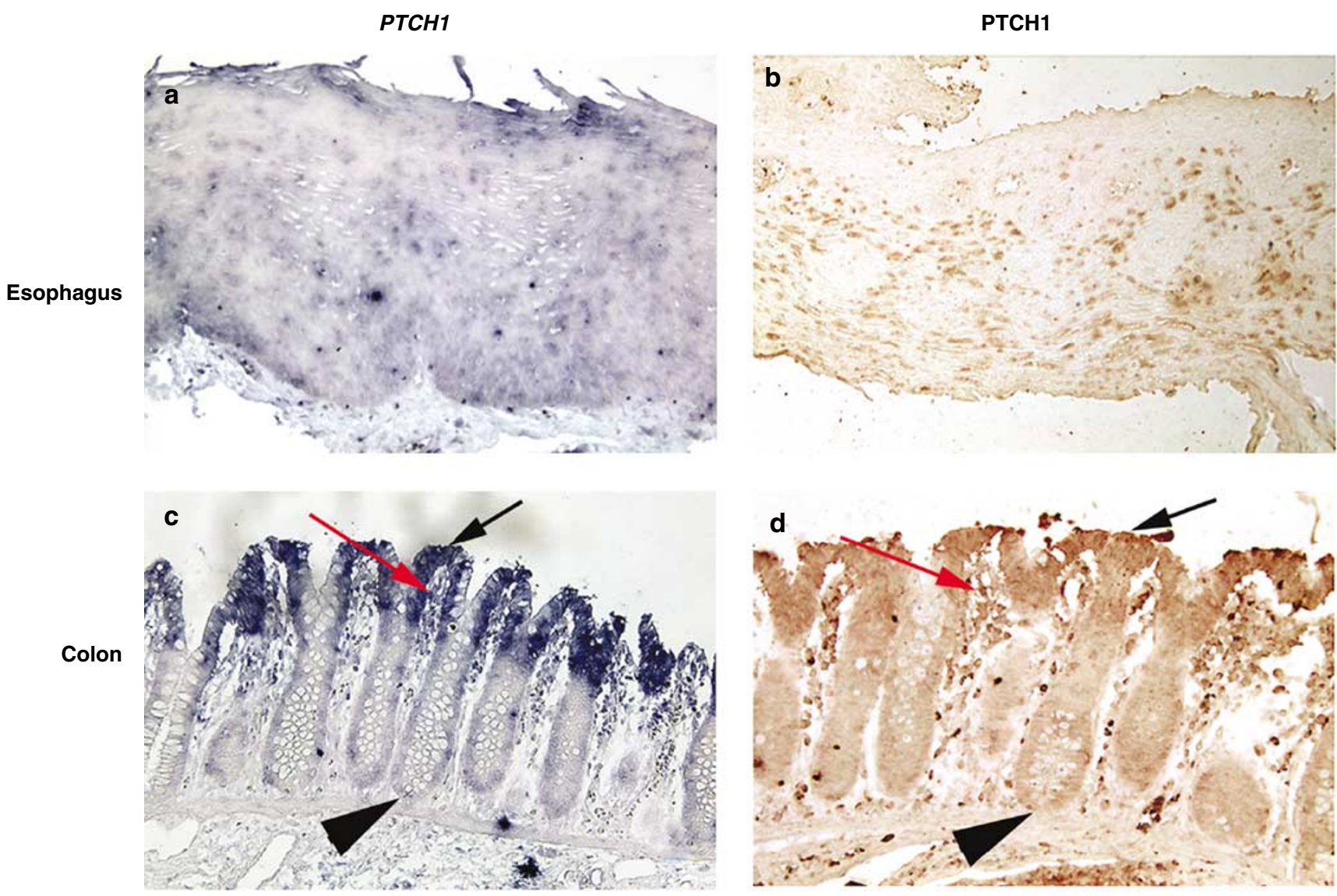

Figure 2 PTCH1 is expressed in normal adult gut. The expression of PTCH1/PTCH1 (mRNA, a and $\mathbf{c}$; protein, b and d) is similar to SHH/ $\mathrm{SHH}$ in normal adult gut and in inflammatory gut in all regions except for the colon. Only background expression is detected in the normal (a, b) esophageal squamous epithelium. The normal stomach expresses PTCH1 in the glands as seen in SHH (data not shown). We did not detect PTCH1/PTCH1 in the lamina propria of any region of the human stomach. Normal small intestine (duodenum, jejunum, and ileum) expresses PTCH1/PTCH1 in the epithelium at base of the villi and in the lamina propria without detectable PTCH1 expression in the villous tips but PTCH1 is present in both epithelial sites (as seen with SHH expression, data not shown). Healthy adult colon expresses PTCH1/PTCH1 in the luminal epithelium (c and d, black arrows) and in the subjacent lamina propria (red arrows) with no expression detected in the crypts (arrowheads). Figure illustrations are limited so as to provide optimal resolution for images shown.

inflamed tissues. We also studied histologically normal biopsies taken from 10 different patients, who were shown not to have inflammatory disease at the time of endoscopy or colonoscopy.

This study confirms our previous report, and shows that the mRNA and protein expression of SHH/SHH are similar. No mRNA or protein expression is detectable in normal squamous epithelium of the esophagus (Figure 1a and b). In the stomach, mRNA and protein (are expressed strongly), and both are restricted to the fundic glands (Figure 1c and d). In the small intestine, SHH mRNA expression is detected only in the epithelium at the villus base (Figure 1e) and in the Brunner's glands of the duodenum (data not shown). However, weak protein expression can be detected in all small intestinal epithelial cells, but is most robust in the epithelium at the base of villi (Figure 1f). Colonic mRNA expression is nearly restricted to the crypts (Figure 1g). Protein expression is strongest in the crypt epithelium, but can also be detected weakly and focally in the surface epithelium (Figure 1h).

Figure 3 Expression of SHH/SHH in inflammatory gut diseases. Strong expression of both mRNA and protein is present in glandular metaplasias in Barrett's esophagitis (arrows in $\mathbf{a}$ and $\mathbf{b}$, and inset a) with adjacent squamous epithelium negative (arrowhead in b). All epithelium gastritis mucosa strongly expresses SHH/SHH (c and d, inset d shows superficial expression, arrow). Similarly all epithelium expresses SHH/SHH in Crohn's disease of the small bowl (e and f, inset $\mathbf{f}$ arrows show surface expression) and in both colonic Crohn's disease (data not shown) and ulcerative colitis (g and two panels in $\mathbf{h}$ ). Arrow in $\mathbf{g}$ and $\mathbf{h}$ highlight luminal epithelial expression. We detect strong expression in inflammatory cells in all inflamed mucosa (red asterisks). 
Inflammatory Gut Disorders

SHH

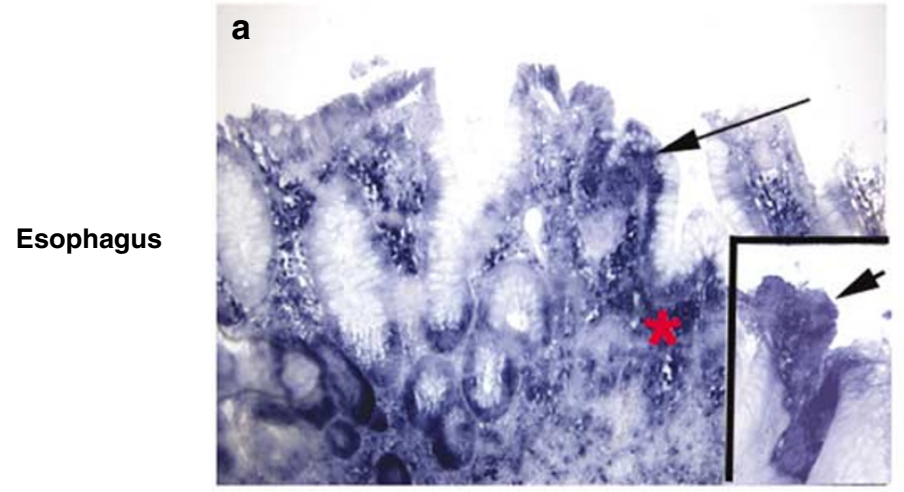

Small Intestine

Colon
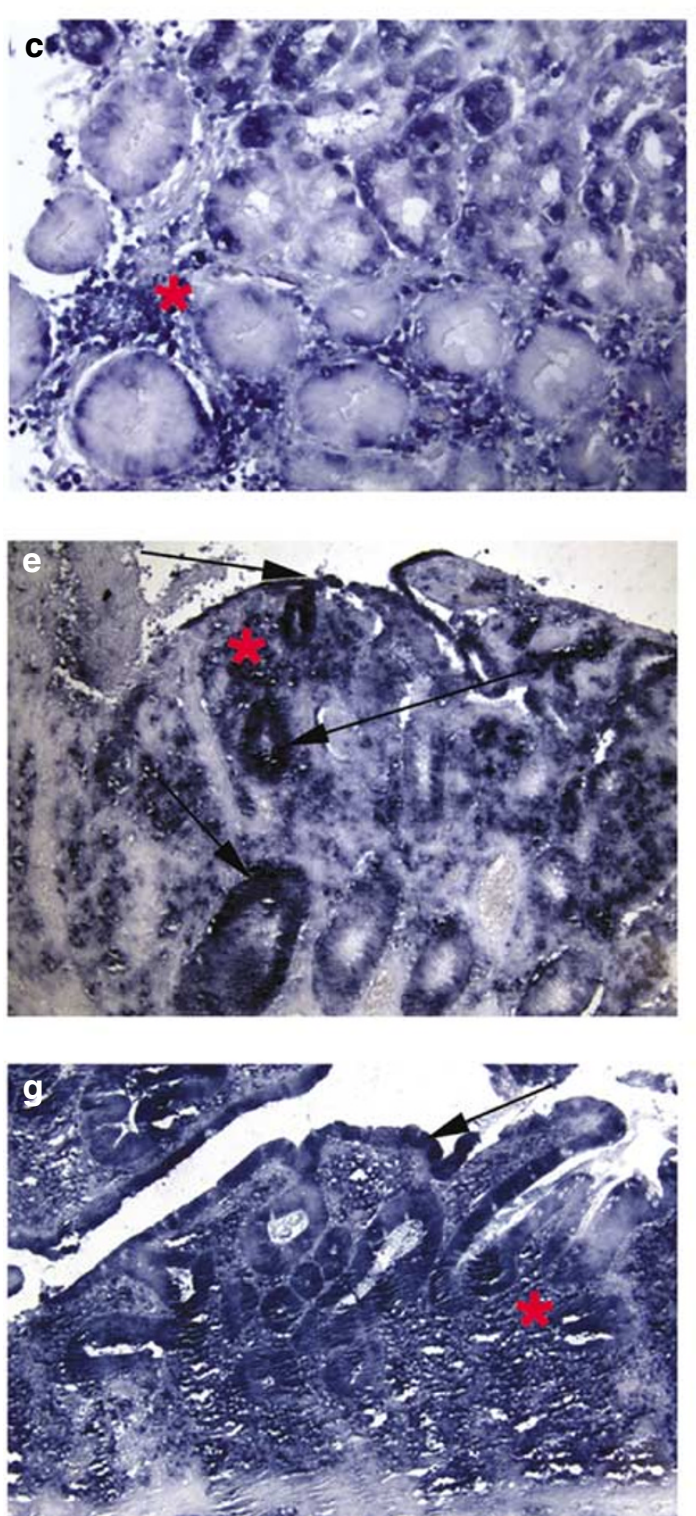

SHH
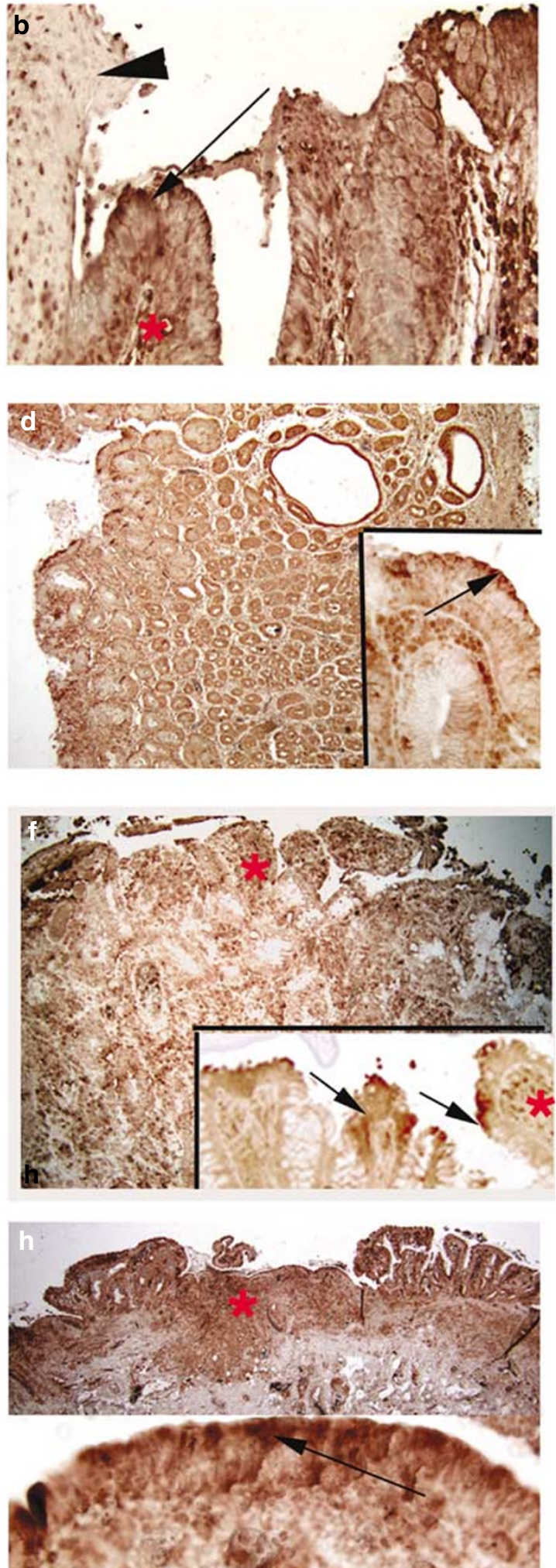
Inflammatory Gut Disorders

PTCH1
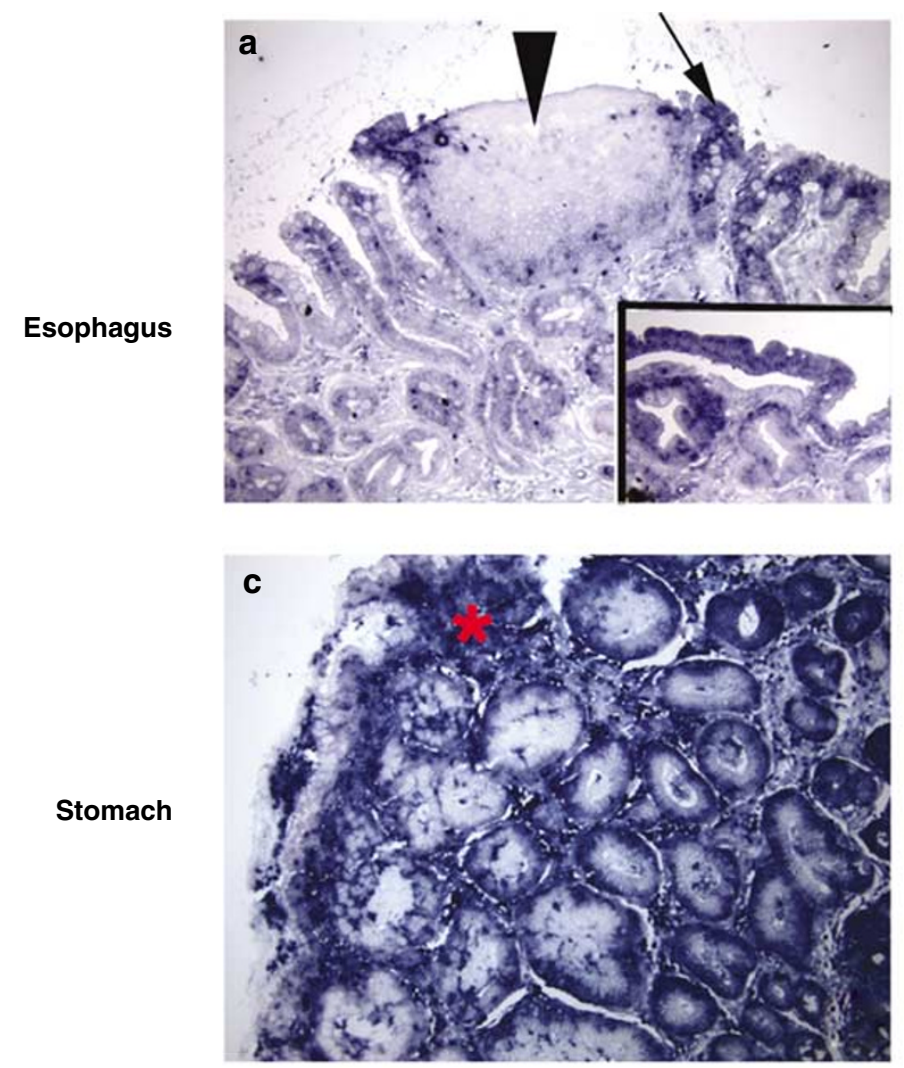

Small Intestine
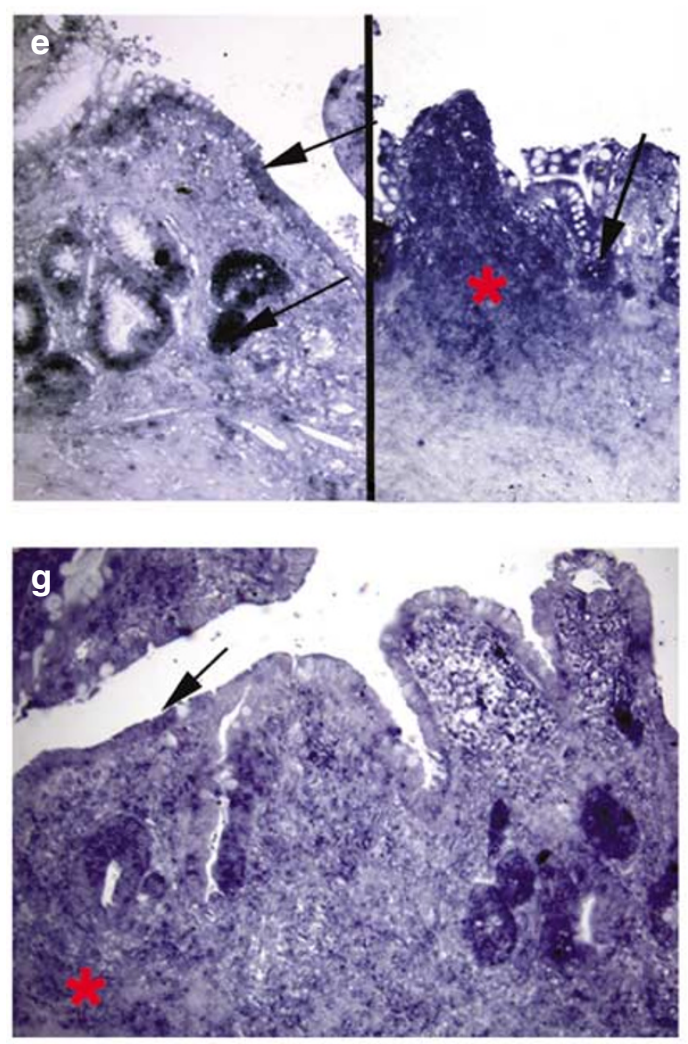

PTCH1
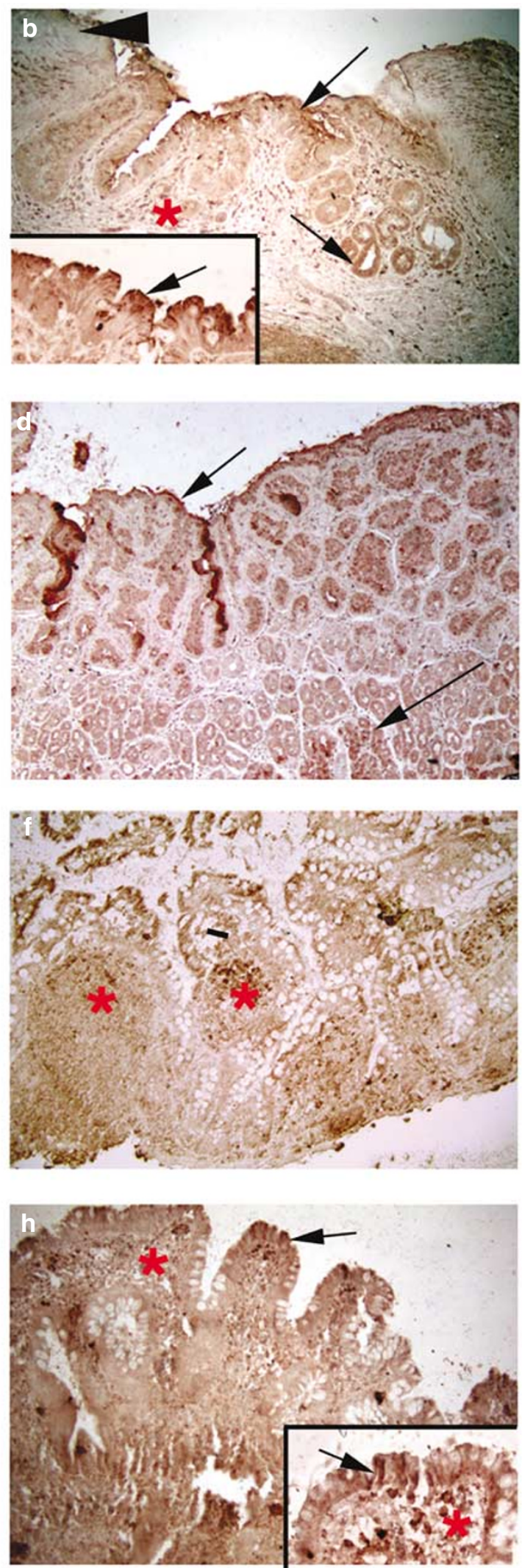
Hence, SHH mRNA expression is spatially restricted within the gut epithelium, sparing the most luminal regions and being present only in glandular (not squamous) epithelium. SHH protein expression follows mRNA expression in the stomach, but differs somewhat in the intestines, with expression in the more superficial epithelium as well. All tissues showed expression in the ganglia of the enteric nervous system (data not shown) and in scattered inflammatory cells in the lamina propria (see below).

\section{PTCH1/PTCH1 Expression in Normal Adult Human Gut}

Similar to SHH/SHH, we did not detect PTCH1/ PTCH1 expression in the esophageal squamous epithelium (Figure 2a and b). PTCH1/PTCH1 expression is present in the fundic glandular epithelium of the stomach and in epithelium in the base of the villi of the small intestine (data not shown). PTCH1/PTCH1 expression is also present in the stromal cells of the lamina propria in both the small intestine and colon with strongest expression in the lamina propria nearest the lumen (red arrows in Figure 2) and in scattered inflammatory cells in the mucosa (see below). The expression of PTCH1/ PTCH1 differs from SHH/SHH in the colonic epithelium. We find both the mRNA and protein expression of PTCH1 restricted in the epithelium to the luminal epithelium (Figure 2c and d) whereas $\mathrm{SHH}$ is expressed weakly in the crypt epithelium and only weakly and patchy in the luminal epithelium (Figure 1g and $\mathrm{h}$ ). In all gut regions, we detect PTCH1/PTCH1 expression in the enteric nervous system ganglia (data not shown).

\section{SHH Expression in Inflammatory Conditions of the Adult Human Gut}

The $S H H / S H H$ expression pattern is altered in human inflammatory gastrointestinal disorders (Figure 3). The normal radial (crypt-villous) pattern of $\mathrm{SHH} / \mathrm{SHH}$ expression is disturbed in inflamed epithelium. Normally, SHH/SHH expression can be detected most strongly and often only in the basal layers of each region of the gut (that thought to be the germative layer, see Figure 1). In the inflamed gut regions, with the exception of the esophageal squamous epithelium, we detect $S H H / S H H$ strong expression throughout the epithelium from the base to the lumen. $\mathrm{SHH} / \mathrm{SHH}$ expression is strongly detected in the metaplastic and inflamed glandular epithelium (both gastric and intestinal metaplasias) in nine of nine cases from different patients with Barrett's esophagus (four associated with adenocarcinoma-resections and biopsies, five with reflux esophagitis-biopsies) (Figure 3a and b). We do not detect $S H H / S H H$ in any of the patients' uninflamed normal squamous esophageal epithelium (data not shown). In all conditions studied, $\mathrm{SHH} / \mathrm{SHH}$ was detected in the inflammatory cells present in the mucosa (Figure 1, red asterisks).

We found the epithelial expression of $\mathrm{SHH} / \mathrm{SHH}$ is diffuse in the other inflammatory conditions studied, including all five separate cases of 'gastritis' (one gastrectomy with gastric adenocarcinomaatrophic, three biopsies: one with $H$. pylori, one 'chemical', one reflux and one atrophic; Figure 3c and d), three of three separate cases of Crohn's disease of the small intestine (Figure 3e and f), 11 of 11 separate cases of ulcerative colitis (Figure 3g and $\mathrm{h}$ ), and five of five separate cases of Crohn's disease of the colon (data not shown). All small intestine and colon tissues were obtained from colectomy specimens. In all cases with regions of unaffected small intestine or colon, the normal pattern of SHH/ SHH expression was demonstrated (Figure 1 and some data not shown).

\section{PTCH1/PTCH1 Expression in Inflamed Tissues of the Gut}

PTCH1/PTCH1 is present in metaplastic and regenerating epithelial cells of inflammatory disorders of the gut including the esophagus, stomach, and small intestine (Figure 4a-f). In ulcerative colitis (Figure $4 \mathrm{~g}$ and $\mathrm{h}$ ) and colonic Crohn's disease (data not shown), we do not detect PTCH1 mRNA, and only focally detect PTCH1 protein (arrows in Figure 4h) in the surface (luminal-most) epithelium. Both PTCH1 mRNA and protein are strongly detected in the crypts. In all regions, PTCH1/PTCH1 is strongly detected in the inflammatory cells in the lamina propria and epithelium (red asterisks in Figure 4).

\section{SHH/SHH and PTCH1/PTCH1 in Midgestation Human Fetal Intestine and Colon}

SHH/SHH expression is present in all epithelial cells of the fetal small intestine but levels are strongest in the base of the villi (arrows in Figure

Figure 4 Expression of PTCH1/PTCH1 in inflammatory gut diseases. Expression of PTCH1/PTCH1 mirrors that of SHH/SHH in all regions of the gut affected with inflammatory diseases. Barrett's esophagitis expressed PTCH1/PTCH1 in all metaplastic glandular epithelium (Figure 1a and $\mathbf{b}$, arrows), sparing squamous epithelium (arrowheads). Inset $\mathbf{a}$ and $\mathbf{b}$ shows higher power of luminal glandular epithelium positive for PTCH1/PTCH1. Gastritis (c and d) and Crohn's disease of the small intestine (e and $\mathbf{f}$ ) show strong expression in all levels of epithelium (arrows). In all cases of colitis studied, we detect PTCH1/PTCH1 in nonsurface epithelium and only patchy expression on the luminal epithelium (ulcerative colitis in $\mathbf{g}, \mathbf{h}$, and inset $\mathbf{h}$, with positive expression highlighted by black arrows). All inflamed mucosa show strong expression of PTCH1/PTCH1 in the inflammatory cells. 


\section{Normal Fetal Tissues}
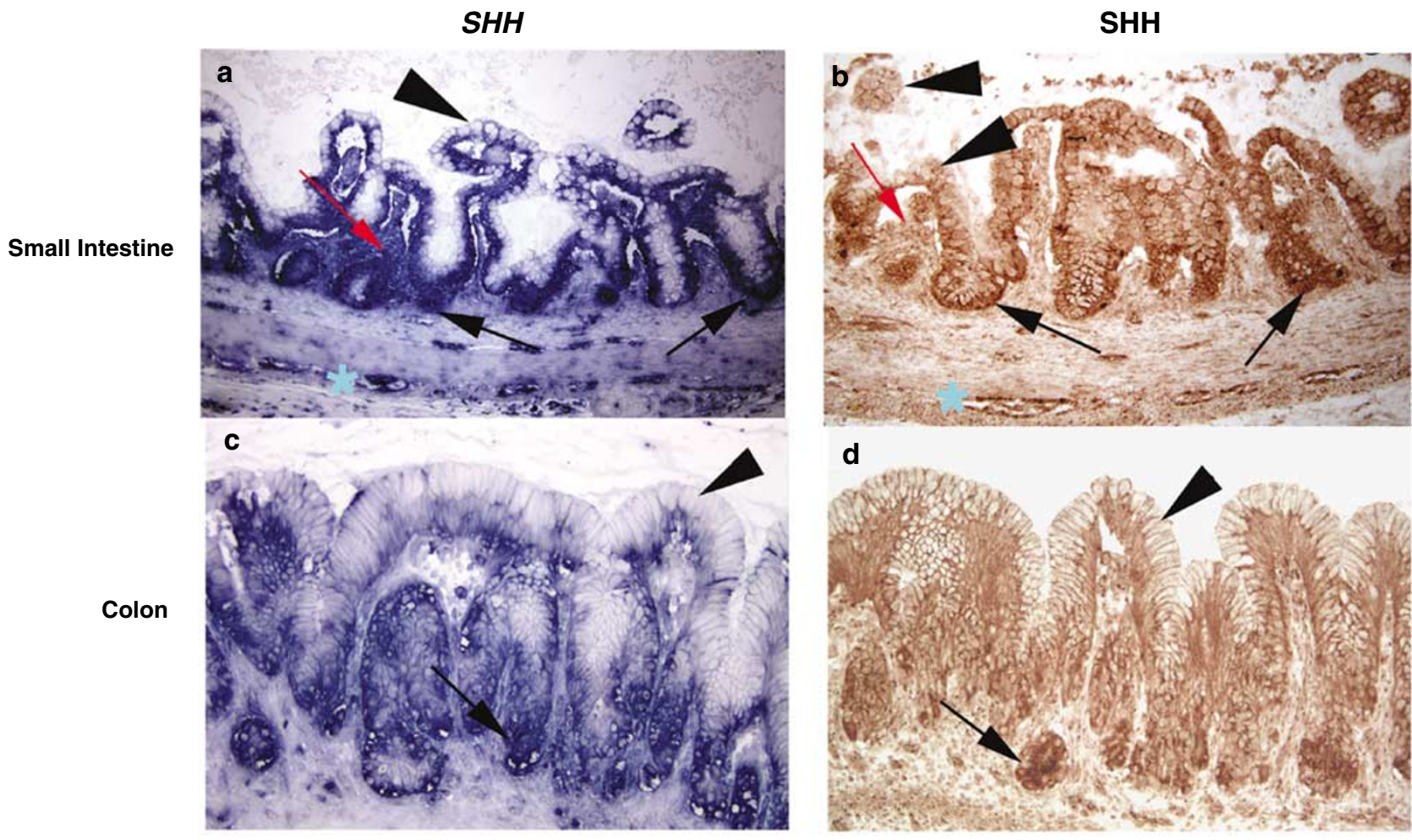

\section{PTCH1}
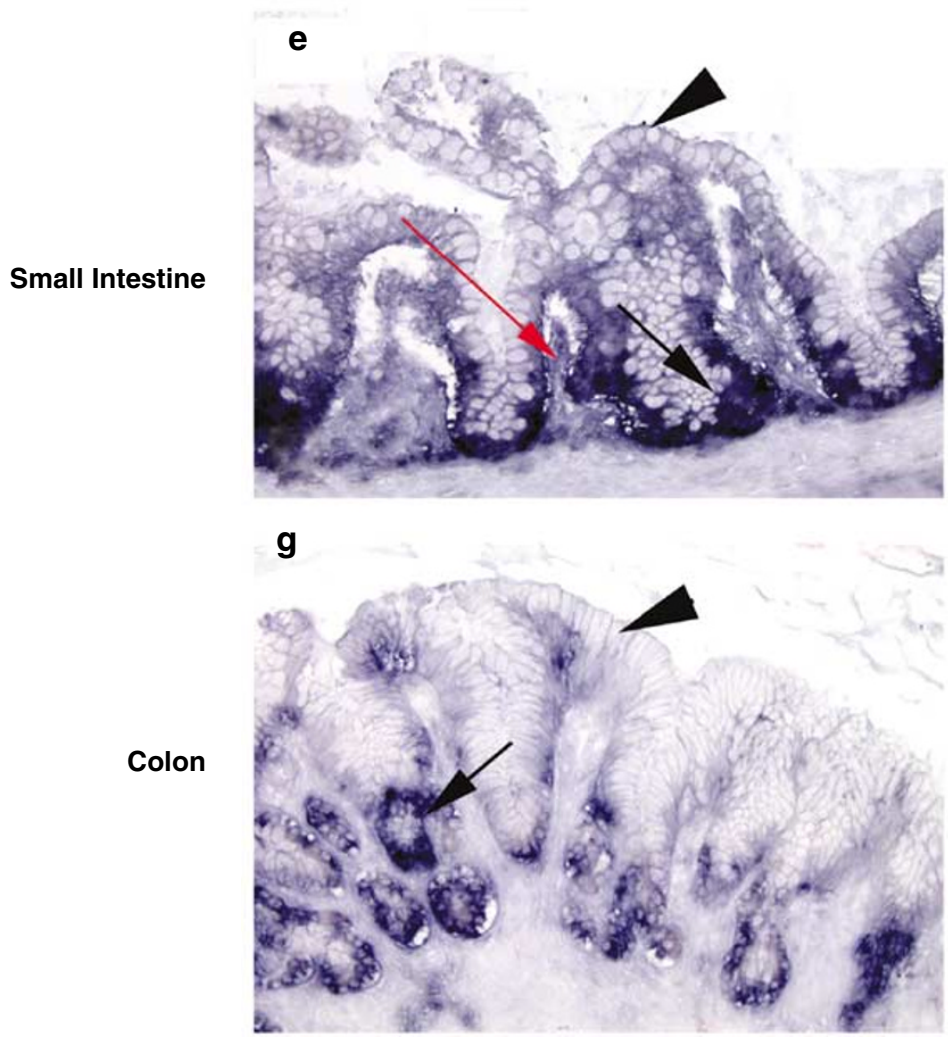

\section{PTCH1}
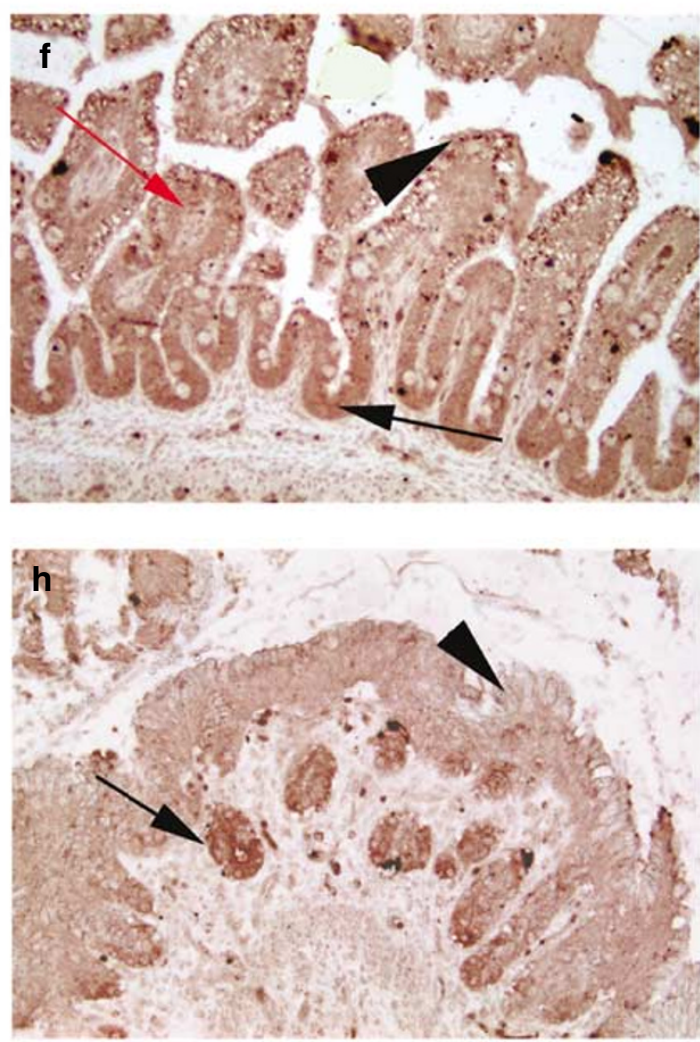
$5 \mathrm{a}$ and b). We also detect strong mRNA (Figure 5a, red arrow) and weaker protein levels (Figure 5b, red arrows) in the small intestinal lamina proprial stroma. Colonic epithelial mRNA expression is restricted to the epithelial crypts as in adults (black arrows Figure 5c) and protein expression is much more restricted to the crypt epithelium than seen in adults (Figure 5d). Expression is present in both colon and small intestine enteric nervous system (eg see blue asterisks in Figure 5a and b).

PTCH1 expression mirrored $\mathrm{SHH}$ in both the small and large intestine (Figure $5 \mathrm{e}-\mathrm{h}$ ). Protein expression of PTCH1 is not detected in the lamina propria at either site.

We also detect mRNA and protein for PTCH1/ PTCH1 and SHH/SHH in the fetal thymus (data not shown).

\section{Gut Mucosal Inflammatory Cells in Inflammatory Diseases Coexpress $\mathrm{SHH}$ and CD4}

In cells expressing $S H H$, B-cell markers CD20 and CD34 are not detected (data not shown). Conversely, T-cell antigen CD4 (helper/inducer T-cell marker) is strongly coexpressed in $\mathrm{SHH}$ expressing inflammatory cells (Figure 6). We do not detect CD8 in SHHexpressing cells (data not shown).

\section{Discussion}

The role of the Hh signaling pathway in adult human gut tissues is not well understood. In other vertebrates, study has focused on its patterning function and the earliest times of gut development. During the early organogenesis period, Shh signaling has been demonstrated to be from endoderm to mesoderm. ${ }^{1,2}$ We show that in human fetal gut at midgestation (much later in development than equivalent chick or murine expression studies), the pathway is primarily active in the epithelium as both ligand and receptor proteins are expressed there (Figure 5). This suggests that there is a change in pathway signaling from epithelial to mesenchymal in early development to a predominantly autocrine signaling within the epithelium in later developmental stages. In adult gut, there may be both as PTCH1/PTCH1 is expressed in the epithelium and the lamina propria (Figure 2).
In intestinal inflammatory gut conditions, expression of these factors is disturbed with a loss of the normal restricted pattern in the radial (crypt-villous) axis. The inflammatory cells in the epithelium and lamina propria express both $\mathrm{SHH} / \mathrm{SHH}$ and PTCH1/ PTCH1 (Figures 3 and 4). These cells are CD4+ lymphocytes (Figure 6). This finding suggests that some of the $\mathrm{Hh}$ signaling may derive from the inflammatory infiltrate in these disorders. Stewart et $a l^{35}$ also found PTCH1 expression in the interstitial infiltrate in a patient with chronic pulmonary fibrosis (see Figure 4e in Stewart et $a^{35}$ ) and found SHH expressed in the affected metaplastic respiratory epithelial cells. They also describe upregulation of Shh expression in a murine model of chronic pulmonary fibrosis and suggest that Shh is required for epithelial repair and maintenance of the inflammatory infiltrate. Whether the signaling is epithelial to inflammatory cell (suggested by Steward et $a l^{35}$ ) or inflammatory cell to epithelium needs to be further investigated. It may be that the expression of Hh proteins outside the epithelium is unrelated to the epithelial expression or its increased expression in injured epithelium. Studies in immunocompromised patients or animal models would be interesting to study this question.

We suggest that the surface SHH protein-expressing cells represent an upregulation of the $\mathrm{SHH}$ protein-expressing stem cells, which normally are spatially restricted to the base of the epithelium. This expression suggests that Hh signaling may play a role in the repopulation of the damaged/altered mucosa by either expansion of the stem cell compartment or inhibition (or delay) of epithelial cell differentiation. This theory provides a logical hypothesis for the neoplastic risk many of these diseases carry. The persistence of stem or undifferentiated cells and abnormal spatial distribution of Hh signaling may predispose the epithelium to malignant transformation, as has been suggested by others. ${ }^{29,47-51}$ Recent publications show strong expression of $\mathrm{SHH} / \mathrm{SHH}$ in malignancies of the gastrointestinal tract including the pancreas, ${ }^{21,23}$ correlating well with our findings of upregulation in preneoplastic diseases of the gut.

While we and others could not detect expression or $\mathrm{SHH}$ in neoplastic colonic epithelium, ${ }^{23,52}$ Oniscu et $a l^{24}$ finds expression upregulated in progression of colonic neoplasia. Herein we describe normal

Figure 5 Expression of $S H H / S H H$ and $P T C H 1 / P T C H 1$ in midgestation human fetal gut tissues. SHH expression is present in the epithelium of the small intestine (a) and colon (c), strongest at the base of the villi or crypts (compare black arrows with arrowheads in a and c). Expression is also present in the lamina proprial cells in the small intestine (red arrow, a). SHH expression mirrors that of $S H H$ (b, d). Expression is also detected in the enteric nervous system (see ganglia marked with blue asterisk in a, b). PTCH1 expression is the same as $\mathrm{SHH}$ in the human fetal small intestine and colon, with strongest epithelial expression in the epithelium at the base of the villi (black arrow in e) or crypts (black arrow in g). The arrowhead shows background expression in the luminal epithelium (e, g). PTCH1/ PTCH1 expression is also present in the lamina propria of the small intestine (red arrows in e and f). PTCH1 expression mirrors PTCH1 expression (f, g) and is also present in the ganglia of the enteric nervous system (data not shown). In the small intestine, PTCH1 expression is also present in the luminal epithelium (arrowhead in $\mathbf{f}$ ) but not in the luminal epithelium of the colon (arrowhead in $\mathbf{h}$ ). 

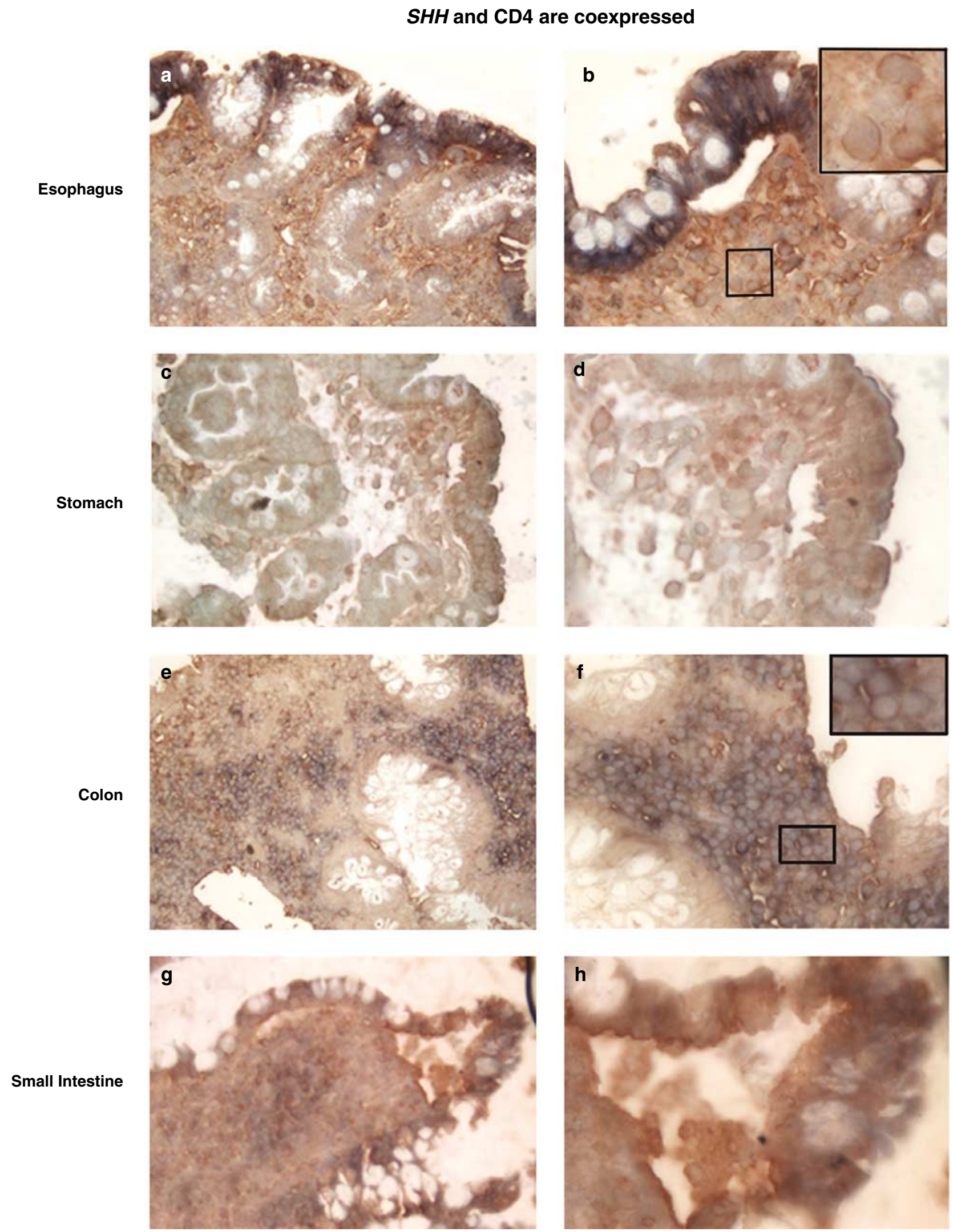

Figure 6 Cells in inflamed gut tissue coexpress SHH and the CD4 antigen. SHH and CD4 expression in metaplastic mucosa of Barrett's esophagitis (a, b), gastritis (c, d), ulcerative colitis (e, f), and Crohn's disease of the ileum (g, h) and colon (data not shown). Brown staining shows CD4 positivity. Insets show blue staining of $S H H$ in CD4-positive cells. 
colonic epithelial expression of SHH mRNA restricted to the crypts (Figure 1) but show that protein expression can be focally (weakly) detected in epithelium even at the surface (luminal). Oniscu et al shows expression only at the lumen by immunohistochemistry for both SHH and PTCH1 (Figure 1a in Oniscu et $a l^{24}$ ) but find mRNA expression in crypts (Figure 2 in Oniscu et $a l^{24}$ ). These discrepancies should be studied further and may only represent different techniques of detection or perhaps different patient ages or regions of the colon.

We do not detect PTCH1/PTCH1 in inflamed colonic surface epithelium and only weakly detect SHH protein. The luminal epithelial location of PTCH1/PTCH1 expression in the normal, uninflamed, adult colon also contrasts with its expression elsewhere in the gut and constrasts with the crypt location of $\mathrm{SHH} / \mathrm{SHH}$ expression. These findings suggest that $\mathrm{Hh}$ signaling may have a different role in colon compared with other regions of the gastrointestinal tract. We have noted expression of $I H H / \mathrm{IHH}$ in the colonic surface most luminal epithelium (similar to that of PTCH1/PTCH1) and therefore IHH may be the functional Hh protein in the human adult colon. Hh signaling in the colon may be more directed towards gut epithelial differentiation as apposed stem cell maintenance elsewhere in the gut. ${ }^{52,53}$ Support for the role of SHH in gut epithelial stem cell maintenance was recently demonstrated when SHH expression was noted to be associated with neoplastic gut diseases. ${ }^{21,23}$ These authors describe SHH expression (protein and mRNA) in epithelial malignancies of the gut and pancreas (but not the colon) in animal models, cell lines, and on human pancreatic tissue sections demonstrating the progression of pancreatic malignancies from dysplasia (in situ) to frank carcinoma. The authors (and we herein) suggest that the abnormal persistence or ectopic expression of $\mathrm{SHH}$ may stimulate an abnormal stem cell hyperplasia, suggested to be a critical step in the neoplastic transition of these tissues.

\section{Acknowledgements}

We thank the Roberts and Lizbeth Perkins laboratories for their comments and hard work. We thank C Tabin for reagents and inspiration. We thank the Department of Pathology at the Massachusetts Hospital especially the members of the gastrointestinal pathology service who assisted in retrieving cases for this study. Supported by the National Institutes of Health (Grant HD34448-03 to DJR).

\section{References}

1 Roberts DJ, Smith DM, Goff DJ, et al. Epithelialmesenchymal signaling during the regionalization of the chick gut. Development 1998;125:2791-2801.
2 Roberts DJ, Johnson RL, Burke AC, et al. Sonic hedgehog is an endodermal signal inducing Bmp-4 and Hox genes during induction and regionalization of the chick hindgut. Development 1995;121: 3163-3174.

3 Matsumoto H, Zhao X, Das SK, et al. Indian hedgehog as a progesterone-responsive factor mediating epithelial-mesenchymal interactions in the mouse uterus. Dev Biol 2002;245:280-290.

4 Walterhouse DO, Lamm ML, Villavicencio E, et al. Emerging roles for hedgehog-patched-Gli signal transduction in reproduction. Biol Reprod 2003;69: 8-14.

5 Fukuda K, Yasugi S. Versatile roles for sonic hedgehog in gut development. J Gastroenterol 2002;37:239-246.

6 Narita T, Ishii Y, Nohno T, et al. Sonic hedgehog expression in developing chicken digestive organs is regulated by epithelial-mesenchymal interactions. Dev Growth Differ 1998;40:67-74.

7 Bitgood MJ, McMahon AP. Hedgehog and Bmp genes are coexpressed at many diverse sites of cell-cell interaction in the mouse embryo. Dev Biol 1995;172: 126-138.

8 Warburton D, Bellusci S, Del Moral PM, et al. Growth factor signaling in lung morphogenetic centers: automaticity, stereotypy and symmetry. Respir Res 2003;4:5.

9 Cardoso WV. Molecular regulation of lung development. Annu Rev Physiol 2001;63:471-494.

10 Stenn KS, Paus R. Controls of hair follicle cycling. Physiol Rev 2001;81:449-494.

11 Warburton D, Zhao J, Berberich MA, et al. Molecular embryology of the lung: then, now, and in the future. Am J Physiol 1999;276(Part 1):L697-L704.

12 Hay ED. An overview of epithelio-mesenchymal transformation. Acta Anat (Basel) 1995;154:8-20.

13 Apelqvist A, Ahlgren U, Edlund H. Sonic hedgehog directs specialised mesoderm differentiation in the intestine and pancreas. Curr Biol 1997;7:801-804.

14 Ramalho-Santos M, Melton DA, McMahon AP. Hedgehog signals regulate multiple aspects of gastrointestinal development. Development 2000;127:2763-2772.

15 Sukegawa A, Narita T, Kameda T, et al. The concentric structure of the developing gut is regulated by Sonic hedgehog derived from endodermal epithelium. Development 2000;127:1971-1980.

16 Ingham PW, McMahon AP. Hedgehog signaling in animal development: paradigms and principles. Genes Dev 2001;15:3059-3087.

17 Taipale J, Beachy PA. The Hedgehog and Wnt signalling pathways in cancer. Nature 2001;411:349-354.

18 Cohen Jr MM. The hedgehog signaling network. Am J Med Genet 2003;123A:5-28.

19 Smith DM, Nielsen C, Tabin CJ, et al. Roles of BMP signaling and $\mathrm{Nk} \times 2.5$ in patterning at the chick midgut-foregut boundary. Development 2000;127: 3671-3681.

20 Hebrok M, Kim SK, Melton DA. Notochord repression of endodermal Sonic hedgehog permits pancreas development. Genes Dev 1998;12:1705-1713.

21 Thayer SP, Di Magliano MP, Heiser PW, et al. Hedgehog is an early and late mediator of pancreatic cancer tumorigenesis. Nature 2003;425:851-856.

22 van den Brink GR, Hardwick JC, Nielsen C, et al. Sonic hedgehog expression correlates with fundic gland differentiation in the adult gastrointestinal tract. Gut 2002;51:628-633. 
23 Berman DM, Karhadkar SS, Maitra A, et al. Widespread requirement for Hedgehog ligand stimulation in growth of digestive tract tumours. Nature 2003;425: 846-851.

24 Oniscu A, James RM, Morris RG, et al. Expression of Sonic hedgehog pathway genes is altered in colonic neoplasia. J Pathol 2004;203:909-917.

25 Quinn AG, Epstein Jr E. Patched, hedgehog, and skin cancer. Methods Mol Biol 2003;222:85-95.

26 Bale AE. Hedgehog signaling and human disease. Annu Rev Genomics Hum Genet 2002;3:47-65.

27 Ruiz i Altaba A, Sanchez P, Dahmane N. Gli and hedgehog in cancer: tumours, embryos and stem cells. Nat Rev Cancer 2002;2:361-372.

28 Wicking C, McGlinn E. The role of hedgehog signalling in tumorigenesis. Cancer Lett 2001;173:1-7.

29 Watkins DN, Berman DM, Burkholder SG, et al. Hedgehog signalling within airway epithelial progenitors and in small-cell lung cancer. Nature 2003;422: 313-317.

30 Parisi MJ, Lin H. The role of the hedgehog/patched signaling pathway in epithelial stem cell proliferation: from fly to human. Cell Res 1998;8:15-21.

31 Rogister B, Ben-Hur T, Dubois-Dalcq M. From neural stem cells to myelinating oligodendrocytes. Mol Cell Neurosci 1999;14:287-300.

32 Franceschi RT. The developmental control of osteoblast-specific gene expression: role of specific transcription factors and the extracellular matrix environment. Crit Rev Oral Biol Med 1999;10:40-57.

33 Clatworthy JP, Subramanian V. Stem cells and the regulation of proliferation, differentiation and patterning in the intestinal epithelium: emerging insights from gene expression patterns, transgenic and gene ablation studies. Mech Dev 2001;101:3-9.

34 Baron M. Induction of embryonic hematopoietic and endothelial stem/progenitor cells by hedgehogmediated signals. Differentiation 2001;68:175-185.

35 Stewart GA, Hoyne GF, Ahmad SA, et al. Expression of the developmental Sonic hedgehog (Shh) signalling pathway is up-regulated in chronic lung fibrosis and the Shh receptor patched 1 is present in circulating T lymphocytes. J Pathol 2003;199:488-495.

36 Agostini C, Semenzato G. Immunology of idiopathic pulmonary fibrosis. Curr Opin Pulm Med 1996;2: 364-369.

37 Selman M, King TE, Pardo A. Idiopathic pulmonary fibrosis: prevailing and evolving hypotheses about its pathogenesis and implications for therapy. Ann Intern Med 2001;134:136-151.

38 Outram SV, Varas A, Pepicelli CV, et al. Hedgehog signaling regulates differentiation from double-negative to double-positive thymocyte. Immunity 2000;13: 187-197.
39 Stewart GA, Lowrey JA, Wakelin SJ, et al. Sonic hedgehog signaling modulates activation of and cytokine production by human peripheral CD4+ T cells. J Immunol 2002;169:5451-5457.

40 Lowrey JA, Stewart GA, Lindey S, et al. Sonic hedgehog promotes cell cycle progression in activated peripheral CD4(+) T lymphocytes. J Immunol 2002; 169:1869-1875.

41 Bhardwaj G, Murdoch B, Wu D, et al. Sonic hedgehog induces the proliferation of primitive human hematopoietic cells via BMP regulation. Nat Immunol 2001;2:172-180.

42 Detmer K, Walker AN, Jenkins TM, et al. Erythroid differentiation in vitro is blocked by cyclopamine, an inhibitor of hedgehog signaling. Blood Cells Mol Dis 2000;26:360-372.

43 DiVito KA, Charette LA, Rimm DL, et al. Long-term preservation of antigenicity on tissue microarrays. Lab Invest 2004;84:1071-1078.

44 de Santa Barbara P, Roberts DJ. Tail gut endoderm and gut/genitourinary/tail development: a new tissuespecific role for Hoxa13. Development 2002;129: 551-561.

45 Marigo V, Roberts DJ, Lee SM, et al. Cloning, expression, and chromosomal location of SHH and IHH: two human homologues of the Drosophila segment polarity gene hedgehog. Genomics 1995;28:44-51.

46 Goodrich LV, Jung D, Higgins KM, et al. Overexpression of ptc1 inhibits induction of Shh target genes and prevents normal patterning in the neural tube. Dev Biol 1999;211:323-334.

47 Watkins DN, Berman DM, Baylin SB. Hedgehog signaling: progenitor phenotype in small-cell lung cancer. Cell Cycle 2003;2:196-198.

48 Ingram WJ, Wicking CA, Grimmond SM, et al. Novel genes regulated by Sonic Hedgehog in pluripotent mesenchymal cells. Oncogene 2002;21:8196-8205.

49 Teh MT, Wong ST, Neill GW, et al. FOXM1 is a downstream target of Gli1 in basal cell carcinomas. Cancer Res 2002;62:4773-4780.

50 Fan H, Oro AE, Scott MP, et al. Induction of basal cell carcinoma features in transgenic human skin expressing Sonic Hedgehog. Nat Med 1997;3:788-792.

51 Dahmane N, Lee J, Robins P, et al. Activation of the transcription factor Gli1 and the Sonic hedgehog signalling pathway in skin tumours. Nature 1997;389: 876-881.

52 van den Brink GR, Bleuming SA, Hardwick JC, et al. Indian Hedgehog is an antagonist of Wnt signaling in colonic epithelial cell differentiation. Nat Genet 2004;36:277-282.

53 de Santa Barbara P, van den Brink GR, Roberts DJ. Development and differentiation of the intestinal epithelium. Cell Mol Life Sci 2003;60:1322-1332. 